\title{
AUTOESTIMA, SATISFACCIÓN CON LA VIDA Y CONDICIONES DE HABITABILIDAD EN ADOLESCENTES ESTUDIANTES DE QUINTO AÑO DE MEDIA. UN ESTUDIO FACTORIAL SEGÚN POBREZA Y SEXO
}

\author{
DAVID TARAZONA ${ }^{1}$
}

\begin{abstract}
RESUMEN
El objetivo del presente estudio fue explorar diferencias en los niveles de autoestima, satisfacción con la vida y condiciones de habitabilidad en adolescentes estudiantes de quinto año de educación secundaria con base en un diseño factorial según género y pobreza. La pobreza se evaluó mediante el tipo de colegio, considerando que el distrito seleccionado muestra grandes brechas económicas entre pobladores pobres y no pobres. Se empleó un cuestionario de autorreporte voluntario y anónimo. Los resultados muestran que (1) respecto a la autoestima, las mujeres de escuela pública tuvieron el menor nivel, los varones de colegio privado, el mayor nivel, finalmente los varones de escuela pública y las mujeres de escuela privada tuvieron un similar nivel intermedio; (2) respecto a la satisfacción con la vida, nuevamente los varones de escuela pública alcanzaron el nivel más alto, las mujeres de colegio privado se ubicaron en segunda posición, y los hombres y mujeres de escuela pública tuvieron un similar nivel bajo; finalmente, (3) las condiciones de habitabilidad fueron mejores en estudiantes de escuela privada y peores en escuela pública, este hallazgo refuerza el uso del tipo de colegio como indicador de pobreza.
\end{abstract}

Palabras clave: Bienestar, autoestima, satisfacción con la vida, pobreza.

\begin{abstract}
The purpose of the present study was to explore the differences in self-esteem, satisfaction with life and habitability conditions in fifth year high school teenager students based in a factorial study by poverty and sex. Poverty was appraised by school type, because there are big breaches between poor and no-poor population in the district selected (La Molina). An anonymously and voluntarily self-reported questionnaire was used. The results shows that (1) about self-esteem, public school women had the smaller level, private school men had the highest and public school men and private school women were in a similar intermediate level; (2) about satisfaction with life, private school men had, again, the highest level, private school women had the second position and public school women and men had the smallest level with similar scores; finally (3) habitability conditions were better in private students and worst in public school students, this find reinforce the use of school type as a poverty indicator.
\end{abstract}

Keywords: Well-being, self-esteem, satisfaction with life, poverty.

1 Instituto Psicología y Desarrollo (IPSIDE). E-mail: david@ipside.org 
La pobreza es el polo opuesto de la riqueza, en términos conceptuales y de estructura social (COSUDE, 2000). A pesar de su carácter multidimensional y complejo, se define en forma precisa al basarse en indicadores de "bienestar", definiéndose operacionalmente como "una condición en la cual una o más personas tienen un nivel de bienestar inferior al mínimo necesario para la sobrevivencia" (INEI, 1999). Este concepto es puntual y de fácil comprensión, pero al fundamentarse en la carencia de bienes materiales resulta siendo relativo por lo que vale complementar su abordaje, considerándola "una situación social en la cual existen carencias económicas, en un tiempo y espacio determinados, que afectan el desarrollo integral del ser humano" (Estefanía \& Tarazona, 2003; p. 22).

La pobreza puede clasificarse como (a) crónica: cuando se transmite generacionalmente, de familia en familia, o (b) temporal: provocada por un retroceso momentáneo que es posible superar (Ardila, 1979). En Psicología se presta mayor atención a la pobreza crónica ya que genera una cultura de subsistencia y porque tiene secuelas psicológicas difíciles de revertir por afectar la infancia temprana, como p.e., la desnutrición crónica y su consecuente influencia negativa en los procesos neuro-cognitivos y la socialización deficitaria y sus efectos en la constitución de una personalidad saludable.

El uso de la variable "tipo de colegio" para estimar la pobreza, es una estrategia habitual en estudios psicológicos y esto en razón de que la mensualidad, escolaridad o pensión es un gasto de consumo familiar establecido en el Método de Líneas de Pobreza, el más popular para evaluar la pobreza a nivel mundial (Tarazona, en prensa). Otra forma similarmente popular de aproximarse a la pobreza es derivada del Método de Necesidades Básicas Insatisfechas y son las condiciones de habitabilidad de las viviendas (Tarazona, en prensa). Cuando estas condiciones son inadecuadas, propician la presencia de enfermedades y trastornos de salud en las familias, especialmente en niños, al punto que se considera que una vivienda inadecuada limita la protección física, intelectual, afectiva y moral de la vida familiar (Instituto de Desarrollo Urbano CENCA, 2001). Sus principales indicadores son hacinamiento, acceso a servicios básicos, estado de la infraestructura y ubicación de la vivienda.

Adolescencia es un término que conlleva un significado psicológico y otro demográfico (FOCUS on Young Adults, 1998). En primera instancia, la adolescencia es un periodo del ciclo vital humano caracterizado principalmente por la aparición de las características sexuales secundarias, una evolución de los procesos psicológicos y modos de identificación del comportamiento y una transición de un estado de total dependencia a uno de dependencia relativa. En segunda instancia, la adolescencia corresponde a toda persona comprendida entre los 10 y 19 años. El grupo más joven, los de 10 a 14 años, son considerados "adolescentes tempranos" y los de 15 a 19, "adolescentes tardíos".

De la observación de las tareas evolutivas a cumplirse en la adolescencia, se deduce que este periodo se define por la consolidación de la individualidad, es decir, por la diferenciación que una persona logre alcanzar frente a los demás. En este proceso de individualización, juegan un importante rol la interacción social, dado que es el contexto natural del desarrollo humano, y la satisfacción de necesidades básicas, estado que le permitirá cumplir estas tareas (SEPEC, 1996). Indudablemente es una etapa que se vería afectada negativamente por condiciones de pobreza o ausencia de bienestar, dado que en la adolescencia, varones y mujeres descubren sus diferencias y a partir de ello configuran su personalidad e identidad (SEPEC, 1996; González Rey, 1999). 
Además de la pobreza, se sabe que el género influye críticamente durante la adolescencia. La adquisición de la identidad sexual y de género se ve influida por muchos cambios físicos, psicológicos y sociales producidos y de los modos de adaptación que los resuelvan (Ferrer, 1994). La interiorización de los roles de género provoca conflictos y diferencias, por un lado a los hombres se les impulsa a definir su identidad en función al éxito sexual, mientras que las mujeres deben conciliar el verse atractivas y, a la vez, reprimirse sexualmente (The Diagram Group, 1981),

La autoestima se refiere a la confianza básica en las propias potencialidades, y apunta a dos componentes esenciales: La valía personal y el sentimiento de capacidad personal (Undurraga \& Avendaño, 1998). La valía personal es la valoración positiva o negativa que la persona tiene de su autoconcepto (imagen de sí mismo), incluyendo las emociones asociadas con esta valoración y las actitudes respecto de sí mismo. El sentimiento de capacidad personal alude a las expectativas que tiene una persona de ser capaz, de hacer de manera exitosa lo que tiene que hacer, es decir, su autoeficacia. En la adolescencia, la autoestima juega un rol muy importante en la configuración de la personalidad y podría verse debilitada, entre otras, por los cambios experimentados en la imagen corporal y la formación propia de una escala de valores y juicios, muchas veces contraria a la de sus padres, generándole inseguridad y sentimientos de ser diferente (FOCUS on Young Adults, 1998).

La satisfacción con la vida es es el componente cognitivo del constructo "bienestar subjetivo" y se define como el sentimiento de bienestar en relación consigo mismo en la propia vida, diferenciándose de la aprobación-desaprobación o el grado de satisfacción con las condiciones objetivas de vida; es básicamente una estimación subjetiva. (Undurraga \& Avendaño, 1998). De otra manera, aunque coincidiendo en su naturaleza, el bienestar subjetivo es concebido como "una evaluación global que la persona hace sobre su vida" (Atienza, Pons, Balaguer \& García-Merita, 2000; p. 314). Las personas que evidencian una alta satisfacción con la vida poseerían una adecuada salud mental, ausente de estrés, depresión, afectos negativos o ansiedad (Atienza, Pons, Balaguer \& García-Merita, 2000).

Si bien existen revisiones sobre las características psicológicas en situaciones de pobreza (Ardila, 1979; Alarcón, 1986; Acevedo, 1996; Estefanía \& Tarazona, 2003), son muy pocas las exploraciones empíricas sobre este tema (p.e. Undurraga \& Avendaño, 1998), mucho menos desde una perspectiva psicosocial o de psicología para el desarrollo, por lo que se vio pertinente llevar a cabo una exploración empírica en población adolescente, dada su vulnerabilidad inherente y el momento que representa en el ciclo vital para la conformación de una personalidad sana, mediante un diseño factorial que permita analizar las interacciones entre sexo y pobreza, a medirse mediante el proxy "tipo de colegio". El objetivo del presente estudio fue explorar diferencias en los niveles de autoestima, satisfacción con la vida y condiciones de habitabilidad en adolescentes estudiantes de quinto año de educación secundaria con base en un diseño factorial según género y pobreza.

\section{MÉTODOS}

\section{Participantes}

La muestra estuvo conformada por 400 estudiantes de quinto año de media matriculados en colegios del distrito de La Molina (51\% varones y $49 \%$ mujeres) y fue proporcional al 
número de estudiantes matriculados en Lima Metropolitana según sexo y tipo de colegio. Del total, 287 provenían de colegios públicos (48.8\% varones y $51.2 \%$ mujeres) y 113 , de colegios privados ( $56.6 \%$ varones y $43.4 \%$ mujeres).

Tabla 1. Participantes según sexo y tipo de colegio.

\begin{tabular}{|c|c|c|c|c|c|c|}
\hline \multirow{3}{*}{ SEXO } & \multicolumn{4}{|c|}{ TIPO DE COLEGIO } & \multirow{2}{*}{\multicolumn{2}{|c|}{ TOTAL }} \\
\hline & \multicolumn{2}{|c|}{ Público } & \multicolumn{2}{|c|}{ Privado } & & \\
\hline & $\mathbf{F}$ & $\%$ & f & $\%$ & $\mathbf{F}$ & $\%$ \\
\hline Varones & 140 & 48.8 & 64 & 56.6 & 204 & 51.0 \\
\hline Mujeres & 147 & 51.2 & 49 & 43.4 & 196 & 49.0 \\
\hline Total & 287 & 100 & 113 & 100 & 400 & 100 \\
\hline
\end{tabular}

A partir de los valores de las variables sexo (varones / mujeres) y tipo de colegio (público / privado), se conformó cuatro grupos que sirvieron para evaluar diferencias en las variables propuestas.

Tabla 2. Grupos en estudio.

\begin{tabular}{|c|c|c|}
\hline GRUPO & SEXO & TIPO DE COLEGIO \\
\hline I & Varones & Público \\
\hline II & Mujeres & Público \\
\hline III & Varones & Privado \\
\hline IV & Mujeres & Privado \\
\hline
\end{tabular}

\section{Materiales e instrumentos}

Se empleó la escala de Autoestima de Rosemberg - modificada, la escala de Satisfacción con la Vida y una escala de Habitabilidad elaborada para este estudio.

Escala de Satisfacción con la Vida (Diener, Emmons, Larsen y Griffin, 1985): Es una escala unidimensional de cinco ítemes positivos que se responden con una escala likert de cinco puntos (Totalmente de acuerdo, de acuerdo, indiferente, en desacuerdo y totalmente en desacuerdo). Para obtener el puntaje directo se suma las respuestas dadas (TA: 5; DA: 4; I: 3; ED: 2, TD: 1).

Escala de Autoestima de Rosemberg - Modificada (Rosemberg, 1965): Consta de ocho ítemes que en su versión original se calificaba como si fuese una escala de tipo Guttman, en este estudio se contestaron y calificaron los ítemes siguiendo el mismo procedimiento que con la Escala de Satisfacción Vital, con la diferencia que en esta se ve la dirección de los ítemes (positiva o negativa). 
Escala de Habitabilidad: Posee diez preguntas redactadas de manera positiva con una forma cerrada de respuesta (SI-NO). Para calcular el puntaje directo se otorga un punto a cada respuesta SI y cero puntos a cada respuesta NO. El onceavo ítem corresponde a la evaluación del hacinamiento obtenida calculando la razón "Número de habitaciones / Número de personas"; en caso la tasa sea mayor a 0.33 (sin hacinamiento) se otorga un punto, de ser menor o igual a 0.33 , se otorga cero puntos.

Tabla 3. Instrumentos empleados.

\begin{tabular}{|c|c|c|}
\hline ESCALA & OBJETIVO & INDICADORES DE CALIDAD \\
\hline $\begin{array}{l}\text { Escala de } \\
\text { autoestima } \\
\text { (Rosemberg) }\end{array}$ & $\begin{array}{l}\text { Conocer la valoración } \\
\text { que una persona hace } \\
\text { con referencia a sí } \\
\text { misma. }\end{array}$ & $\begin{array}{l}\text { Confiabilidad: Alta } \\
\text { Alpha de Cronbach (Consistencia interna) }=0.77 \\
\text { Validez: Alta (\# de factores }=2 \text { ) } \\
\% \text { de la varianza explicada }=53.07 \\
\% \text { de la v. e. por el primer factor }=27.98\end{array}$ \\
\hline $\begin{array}{l}\text { Escala de } \\
\text { satisfacción con } \\
\text { la vida (SWLS) }\end{array}$ & $\begin{array}{l}\text { Conocer el grado de } \\
\text { satisfacción de una } \\
\text { persona con su vida. }\end{array}$ & $\begin{array}{l}\text { Confiabilidad: Alta } \\
\text { Alpha de Cronbach (Consistencia interna) }=0.76 \\
\text { Validez: Alta (\# de factores }=1 \text { ) } \\
\% \text { de la varianza explicada }=52.32\end{array}$ \\
\hline $\begin{array}{l}\text { Escala de } \\
\text { habitabilidad }\end{array}$ & $\begin{array}{l}\text { Conocer las condiciones } \\
\text { materiales de la } \\
\text { vivienda. }\end{array}$ & $\begin{array}{l}\text { Confiabilidad: Alta } \\
\text { Alpha de Cronbach (Consistencia interna) }=0.76 \\
\text { Validez: Alta (\# de factores }=3 \text { ) } \\
\% \text { de la varianza explicada }=50.872 \\
\% \text { de la v. e. por el primer factor }=22.47\end{array}$ \\
\hline
\end{tabular}

\section{Procedimientos}

El cuestionario fue aplicado de manera anónima y voluntaria en tres colegios públicos y seis colegios privados del distrito de La Molina. Los colegios públicos estuvieron ubicados en las localidades Las Viñas, Covima y Musa (Un colegio en cada zona), mientras que los colegios privados se encontraban en Las Viñas (3 colegios), Mayorazgo, Santa Patricia y el Sol de la Molina (Un colegio en cada zona). El costo mensual mínimo en colegios privados fue de S/. 190 y el máximo fue $S /$. 540, en tanto el costo en colegios públicos es subvencionado por el Estado. Culminado el trabajo de campo se procedió a descartar los cuestionarios inválidos y luego se elaboró una base de datos en el programa SPSS v. 10 para poder efectuar el procesamiento estadístico.

\section{RESULTADOS}

En la Tabla 4 se aprecia que en las tres variables medidas se observan diferencias significativas entre los grupos de estudio siendo más intensa en el caso de la habitabilidad. 
Tabla 4. Diferencias entre grupos.

\begin{tabular}{|c|c|c|c|c|c|c|}
\hline \multirow{2}{*}{ GRUPOS } & \multicolumn{2}{|c|}{ AUTOESTIMA } & \multicolumn{2}{c|}{$\begin{array}{c}\text { SATSFACCIÓN CON } \\
\text { LA VIDA }\end{array}$} & $\begin{array}{c}\text { CONDICIONES DE } \\
\text { HABITABILIDAD }\end{array}$ \\
\cline { 2 - 7 } & Media & $\begin{array}{c}\text { Media en } \\
\text { escala \% }\end{array}$ & Media & $\begin{array}{c}\text { Media en } \\
\text { escala \% }\end{array}$ & Media & $\begin{array}{c}\text { Media en } \\
\text { escala \% }\end{array}$ \\
\hline I & 31.36 & 78.40 & 15.67 & 62.68 & 7.09 & 64.45 \\
\hline II & 30.42 & 76.05 & 15.79 & 63.16 & 7.29 & 66.27 \\
\hline III & 32.84 & 82.10 & 17.45 & 69.80 & 8.84 & 80.36 \\
\hline IV & 31.33 & 78.33 & 16.47 & 65.88 & 9.10 & 82.73 \\
\hline Rango & \multicolumn{2}{|c|}{$0-40$} & \multicolumn{2}{c|}{$0-25$} & \multicolumn{2}{c|}{$0-11$} \\
\hline \multirow{2}{*}{ ANOVA } & \multicolumn{2}{|c|}{$\mathrm{F}=3.85$} & \multicolumn{2}{c|}{$\mathrm{F}=3.28$} & \multicolumn{2}{c|}{$\mathrm{F}=19.21$} \\
\cline { 2 - 7 } & \multicolumn{2}{|c|}{$\mathrm{p}<0.01$} & \multicolumn{2}{c|}{$\mathrm{p}<0.02$} & \multicolumn{2}{c|}{} \\
\hline
\end{tabular}

Observando el Gráfico 1, se aprecia que los niveles de autoestima y satisfacción con la vida varían en un rango similar, en tanto que las condiciones de habitabilidad sí muestran una marcada pendiente entre los grupos de colegios público y privado, lo que lo que guarda coherencia con la mayor intensidad observada en la Tabla 1. Todos los puntajes están por encima de los valores medios posibles.

Gráfico 1. Autoestima, satisfacción con la vida y habitabilidad según los grupos estudiados.

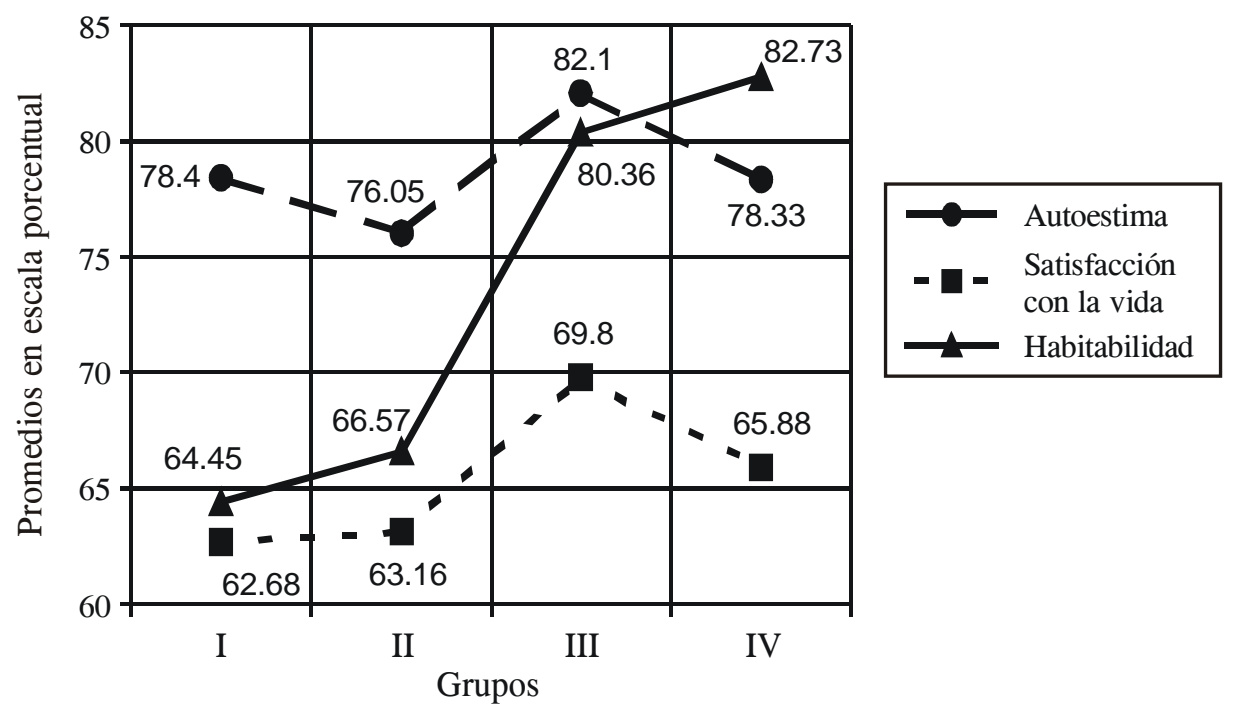


Los niveles de autoestima conformarían tres conjuntos sobre la base de la diferencia significativa entre los cuatro grupos, establecida por análisis de varianza de una sola vía $(\mathrm{F}=3.85 ; \mathrm{p}<0.01)$. Primero, el mayor nivel corresponde a los varones de colegio privado (GIII). Segundo, los varones de colegios estatales (GI) y las mujeres de colegio privado (GIV). Finalmente, en tercera locación se ubicarían las mujeres de colegio público (GII).

En lo que respecta al nivel de satisfacción con la vida, nuevamente se conformarían tres grupos con base en una diferencia significativa hallada en el análisis de varianza $(F=3.28$; $\mathrm{p}<0.02$ ). El primer lugar estaría ocupado por los varones de colegio privado (GIII); el segundo, por las mujeres de colegio privado (GIV); y el tercero, por mujeres (GI) y varones (GII) de colegio público. Obsérvese que en esta variable, los grupos de colegio privado toman las posiciones más altas, en tanto que los de escuelas públicas, los más bajos.

Las condiciones de habitabilidad conforman dos conjuntos. Primero, son más altas en estudiantes de colegio privado; segundo, son más bajas en sus pares de colegio público. En ambos casos, las mujeres ocupan los lugares más altos. La significación estadística de la diferencia hallada fue establecida tanto al hacerse el análisis de varianza entre los cuatro grupos conformados $(\mathrm{F}=19.21, \mathrm{p}<0.001)$ como al evaluarse la diferencia de medias entre colegios públicos y privados $(\mathrm{t}=-9.92, \mathrm{p}<0.001)$.

\section{DISCUSIÓN}

Los niveles de autoestima, satisfacción con la vida y condiciones de habitabilidad estarían por encima del promedio teórico, por ello las diferencias entre grupos se darían en el sentido de "mayor desarrollo". Asimismo, en relación con lo anterior, se aprecia que los puntajes en autoestima y satisfacción con la vida varían en un rango similar, en tanto que las condiciones de habitabilidad muestran una marcada pendiente entre los grupos de colegios público y privado por lo que podría sugerirse que (1) las desigualdades socioeconómicas son mayores en términos demográficos que psicológicos e involucrarían en niveles similares a varones y mujeres, y (2) debido a una socialización diferenciada, las mujeres de ambos grupos reportarían mejores condiciones porque son más sensibles a las condiciones del hogar o porque reciben más atención y mejores condiciones de vida por parte de sus familias.

El sexo tendría una influencia importante en interacción con la situación económica sobre los niveles de autoestima. Los varones de colegio privado ocupan la primera posición y las mujeres de colegio público, la última; en tanto los varones de escuela pública y las mujeres de colegio privado obtienen puntajes similares, conformando un grupo intermedio en el que ambos ocupan un mismo puesto sin ser posible definir alguna jerarquía, aunque cabe señalar que los varones de colegio público obtienen un puntaje ligeramente más alto. Por un lado, este hallazgo sugeriría como hipótesis para futuros estudios si es que la autoestima en varones de colegios públicos es mayor que las mujeres de colegios privados, con lo que se podría asumir que la autoestima se ve afectada por diferencias genéricas; y por otro, concordaría con el estudio de Undurraga \& Avendaño (1998), con población chilena, quienes no hallaron diferencias en autoestima entre personas pobres, de capas medias o ricas, resultando todos con una alta valoración de sí mismos en diversos roles sociales (trabajadores, madres / padres y pareja), pero si se halló que al evaluarse diferencias según género, las mujeres mostraron niveles más bajos (Avendaño, 2000). 
Una diferencia más clara, según el tipo de colegio, es la que se evidencia en los niveles de satisfacción por la vida. En este caso, nuevamente los varones de colegio privado ocupan la primera posición, seguidos por las mujeres del mismo tipo de colegio; la última ubicación es ocupada por varones y mujeres de colegio público, con una diferencia ligera a favor de las mujeres. Las diferencias son más definidas que en el caso de la autoestima, pero no se logra tanta claridad como en las condiciones de habitabilidad; en otro aspecto, los niveles alcanzados se ubican por debajo de los niveles de autoestima y de los puntajes más altos de habitabilidad, sugiriendo que, en general, los adolescentes participantes podrían no tener un alto nivel de bienestar subjetivo. El estudio con población chilena de Undurraga \& Avendaño (1998) coincide en el hecho que los pobres se ubiquen en último lugar, pero no conque los niveles sean relativamente bajos independientemente del nivel socioeconómico. Este punto también es divergente de lo hallado con población española por Clemente, Molero \& González (2000), quienes encontraron una muy alta satisfacción en adolescentes y jóvenes, y marca grandes diferencias con los planteamientos de Ardila (1979) y Alarcón (1986).

¿Por qué se han producido estos vínculos? Podemos ensayar tres respuestas: (1) En cuanto a las diferencias entre varones y mujeres, se corrobora que el género influye en los rasgos psicológicos de forma diferencial durante la adolescencia (Ferrer, 1994); asimismo la interacción entre condiciones de vida y socialización diferenciada según género (Mansilla, $\mathrm{s} / \mathrm{f}$ ); (2) en lo que respecta a la mayor definición de las diferencias en satisfacción vital según tipo de colegio, cabe suponer que al ser el componente cognitivo del bienestar subjetivo el resultado estaría reflejando la mayor sensibilidad de la variable para discriminar diferencias y similitudes en la calidad de vida (Arenas, 2003).

Concluimos señalando que la exploración mostrada permite aportar evidencia empírica respecto al efecto negativo de la pobreza en el desarrollo personal, con algo más de intensidad en las mujeres que en los varones.

\section{REFERENCIAS BIBLIOGRÁFICAS}

1. Acevedo, M. I. (1996). Factores de vulnerabilidad asociados a la condición de pobreza. Un enfoque ecosistémico cognitivo. Aprendizaje y comportamiento. 11(1-2), 53-72.

2. Alarcón, R. (1986). Psicología, pobreza y subdesarrollo. Lima: INIDE.

3. Aldana, C. (1995) Fragmentación, desesperanza y humor. Retos al trabajo psicológico con adolescentes de tugurio. pp. 20(131), 61-70.

4. Ardila, R. (1979). "Psicología social de la pobreza". En J. Whittaker (Ed.) La psicología social en el mundo de hoy. México: Trillas.

5. Arenas, C. (2003). "Psicología comunitaria y calidad de vida en el Perú". Revista de Psicología de la UNMSM. VI(2), 99-110.

6. Atienza, F. L.; Pons, D.; Balaguer, I. \& García-Merita, M. (2000). "Propiedades psicométricas de la escala de satisfacción con la vida en adolescentes". Psicothema, 12(2), 314-319. 
7. Avendaño, C. (2000). "Aspectos psicosociales de la pobreza". En VI Seminario internacional. Psicología de la Salud. Lima: Universidad San Martín de Porres.

8. Clemente, A.; Molero R. \& González F. (2000). "Estudio de la satisfacción personal según la edad de las personas". Anales de psicología. 16(2), pp. 189-198.

9. COSUDE (2000). "Política de desarrollo social de COSUDE". En: Respecto a la dificultad de erradicar la pobreza en el mundo. pp. 14-60, Suiza: El autor.

10. Diagram Group, The (1981). El sexo. Manual práctico ilustrado para la relación sexual. España: Everest.

11. Estefanía, M. T. \& Tarazona, D. (2003). "Psicología y pobreza. ¿Hay algo psicológico en la pobreza o es la pobreza algo psicológico?" Explorando psicología. 12, 21-25.

12. Ferrer, M. (1994). "El género en la adolescencia". En Aguirre, A. (Ed.) Psicología de la adolescencia (pp. 77-93). Madrid: Pirámide.

13. FOCUS on Young Adults (1998). Manual de capacitación para facilitadores. Mejorando habilidades y destrezas de comunicación interpersonal para la orientación a adolescentes en salud sexual y reproductiva. Lima: El Autor.

14. González Rey, A. (1999). Comunicación, personalidad y desarrollo. Cuba: Pueblo y Educación.

15. Instituto de Desarrollo Urbano CENCA (2001). Situación general de la vivienda en el Perú. Documento elaborado para la VI Conferencia Nacional sobre Desarrollo Social.

16. INEI (1999). Métodos de medición de la pobreza. Lima: INEI.

17. Mansilla, M. E. (s/f). La socialización diferenciada por género. Lima: CONCYTEC.

18. SEPEC. (1996). "Estoy aquí, queriéndote". Los jóvenes, el amor y la sexualidad. Lima: SEPEC.

19. Tarazona, D. (en prensa) "Psicología y lucha contra la pobreza: Revisión y propuestas desde la psicología social-comunitaria". En Psicología comunitaria. Lima: Foro Peruano de Psicología Social.

20. Undurraga, C. \& Avendaño, C. (1998). "Dimensión psicológica de la pobreza". Psykhe, 6(1), 57-63. 